\title{
A collision term valid from rarefied to dense gases and plasmas
}

\author{
C. J. Z A M L U T T I \\ Instituto Nacional de Pesquisas Espaciais, INPE C.P. 515, \\ 12245-970 São José dos Campos, SP, Brazil \\ (zamlutti@ltid.inpe.br)
}

(Received 20 August 2003)

\begin{abstract}
The Boltzmann collision integral for binary encounters is restricted to rarefied gases and plasmas by the constraint that the mean free path be much larger than the range of interaction. For dense media the two lengths become comparable and a proper account of the mutual influence exerted by one particle on the other during a collision must be taken. There are also other situations involving chargedcharged long-range interactions for which this condition is met even in rarefied plasmas. The matter is considered in this work with the solution of the BBGKY (Born, Bogoliubov, Kirkwood, (H. S.) Green, Yvon) equation to determine the joint distribution function for the encountering particles. It is shown, in particular, that for dense gases the results replace the traditional Enskog $\chi$ factor, whereas for plasmas oscillatory phenomena can be driven. The proposed theory constitutes an improvement over the revised Enskog theory (RET) to the extent that it is not restricted to the soft sphere encounters, but valid for any sort of field interaction between colliding particles. Moreover the correlation term replaces the unsuitable restitution coefficient of the RET approach, which is restricted to impact theory. The present material is important for studies in solar and terrestrial physics, which require the sole knowledge of the one particle distribution function, namely gas dynamics and Coulomb plasma research.
\end{abstract}

\section{Introduction}

Collision theory encloses a wide range of situations and methods to model them. They start with the simplicity of the impact theory of classical mechanics (Landau and Lifchitz 1966) and run to the complexity of the Liouville (Klimontovich 1982), Liouville-von Neumann (Burghardt and Cederbaum 2001) or Heisenberg (Wan and Schofield 2000) and Liouville diffusion equations (Xing 1998), employed to dense gases, plasmas and the reactive interactions of quantum mechanics. The Boltzmann collision term developed for rarefied gases (e.g. Chapman and Cowling 1970; Liboff 1969) provides a satisfactory base for the description of non-equilibrium properties. It was constrained by two severe limitations:

(a) the mean free path be much larger than the interaction range;

(b) the particle integrity be preserved.

The first restriction excludes dense gases (which reduce the mean free path) and plasmas (since Coulomb collisions are long-range interactions). Reactive collisions 
are excluded by condition (b) provided that they modify the particle integrity. For dense gases the Boltzmann term was adjusted by the Enskog $\chi$-factor (Chapman and Cowling 1970). For plasmas it was modified to become the Fokker-Planck term (see the review by Zamlutti (1999)). To deal with reactive interaction, the concepts of semi-classical mechanics were incorporated (Zamlutti 1997). The correlation term described here constitutes an alternative stepping stone towards the solution for restriction (a).

The correlation term is so called because it accounts for what happens to the moving particle when it is under the field effect due to the target particle. Its importance has been stressed in the last four decades (Frieman and Book 1963; Montgomery and Tidman 1964; Liboff 1969; Chapman and Cowling 1970; van Beyeren and Ernst 1979; Klimontovich 1982; Zubarev et al. 1991; Xing 1998) and is estimated by either of the ratios

$$
\tau=t_{\mathrm{c}} / t_{\mathrm{m}}, \quad \chi=\sigma / l_{\mathrm{m}}
$$

where $t_{\mathrm{c}}$ is the time spent within the field effect of the target, $\sigma$ is the radius of the cross section for interaction, $l_{\mathrm{m}}$ and $t_{\mathrm{m}}$ the mean free path and mean collision time. When $\tau \ll 1$ and $\chi \ll 1$, the Boltzmann collision term is valid; otherwise, correlation aspects must be considered.

Since the Liouville approach is equivalent to the Newtonian formalism (Liboff 1969) we start with these equations of motion.

The motion of an individual (bullet) particle of either a gas or plasma can be described by a six-dimensional map (finite difference scheme) as

$$
\begin{gathered}
\mathbf{r}_{i+1}=\mathbf{r}_{i}+h \mathbf{v}_{i} \\
\mathbf{v}_{i+1}=\lambda \mathbf{v}_{i}+h\left(\mathbf{a}_{i}+\mathbf{s}_{i}\right)
\end{gathered}
$$

where $\mathbf{r}$ represents the particle coordinates, $\mathbf{v}$ its velocity, a the force per unit mass acting on it, $\mathbf{s}$ a collisional noise effect, $\lambda=1-\beta h$ with $\beta$ as a collisional transfer coefficient and $h$ the time step, usually $t_{\mathrm{m}}$ for rarefied fluids (see Jensen and Oberman 1982). Bold symbols denote vector entities and the same non-bold symbols are their magnitudes. Initial conditions complement the description.

The collisional noise has the form $\mathbf{s}_{i}=\beta \mathbf{v}_{1 i}+\mathbf{s}_{i}$, where $\mathbf{v}_{1}$ denotes the instantaneous velocity of the colliding target particle and $\mathbf{s}$ represents an actual random noise. If $a \gg s$ we have a field controlled motion. When $\mathbf{s}$ becomes comparable to a the scattering increases reducing the effectiveness of field actions.

By inspection, we see that the action of $\beta$ in $\lambda$ is to develop a functional reaction to the motion. As for its influence on the performance of a on the trajectory, it amounts to introducing a scattering component which destroys the cause-effect coherence.

Two variable maps (phase space maps) were analyzed in detail by Jensen (1987) and Ott (1981) for the case of one dimension. The fact that the above map is threedimensional just extends rather than restricts their results. Then the motion may present a seemingly random behavior when:

(a) the initial conditions are unpredictable (classical statistical mechanics);

(b) there are nonlinearities in either of the evolution terms (chaotic evolution).

The first situation corresponds to the regular behavior of the phenomena whereas the second instance is related to the development of turbulence. A natural 
occurrence of the first case appears in solar-terrestrial physics in the solar wind (Marsch and Livi 1987; Lie-Svendsen and Olsen 1998) and upper terrestrial atmosphere (lower $\beta$ ). As for the second case, it is characteristic of the solar corona and terrestrial lower atmosphere (higher $\beta$ ).

Regardless of the condition for its onset, the statistical feature requires a probabilistic approach to describe the complexity of its dynamics (see Jensen and Oberman 1982). The difficulty increases when the density of the target medium is enhanced or we deal with long-range interactions. In this case, the $h$ of $(1)$ and (2) must be replaced by $h^{*}=t_{\mathrm{m}}-t_{\mathrm{c}}$.

The correlation range and time are related by $\sigma=w t_{\mathrm{c}}$, where $w$ is the magnitude of relative velocity $\mathbf{w}=\mathbf{v}-\mathbf{v}_{1}$ (see Klimontovich (1982) and references therein).

The importance of the correlation term can be evaluated by the ratio

$$
\chi=\sigma / l_{\mathrm{m}} .
$$

The mean free path $l_{\mathrm{m}}$ is evaluated by $l_{\mathrm{m}}=w / v_{1}$ (Bird 1994), where $v_{1}$ is the average collision frequency (see Zamlutti 1994; $\mathrm{Hu}$ and Mei 1998; Collins and Sundaram 1998) and $w$ is the relative velocity during the encounter.

It then results that

$$
\chi=v_{1} \sigma / w=n_{1} \pi \sigma^{3}
$$

since $v_{1}=n_{1} w \pi \sigma^{2}$ (see Zamlutti 1994) and $n_{1}$ is the target number density.

The cross section for moment transfer, $S$, is usually computed (Hirschfelder et al. 1954; Burgers 1969; Schunk 1977; Zamlutti 1994, 1999; Bird 1994) and therefore $\sigma=(S / \pi)^{1 / 2}$. For consistency, we consider the momentum transfer cross-section, $S_{1}$, and assume rotational symmetry.

The following are radii of the sphere of interaction results:

$$
\begin{aligned}
\sigma & =2.0 \times 10^{-8} \mathrm{~cm} \text { (neutral-neutral collisions) } \\
\sigma & =1.5 \times 10^{-5} w^{-1 / 2} \mathrm{~cm}(\text { ion-neutral collisions) } \\
\sigma & =0.25 \times 10^{-8} T^{1 / 4} \mathrm{~cm}(\text { electron-neutral collisions) } \\
\sigma & =0.707\left(\lambda_{\mathrm{D}} / b\right)\left[\ln \left(1+4 b^{2}\right)\right]^{1 / 2} \\
& =7.88 \times 10^{-4} T^{-1}\left[\ln \left(1+4 b^{2}\right)\right]^{1 / 2} \mathrm{~cm}(\text { charged-charged collisions) }
\end{aligned}
$$

where $T$ is the absolute temperature in Kelvin, $k$ is the Boltzmann constant in esu, $e$ is the electron charge in esu, $\lambda_{\mathrm{D}}=690\left(T / n_{1}\right)^{1 / 2}$ is the Debye length in centimetres and $b$ is the ratio of kinetic to potential energy.

The quantity $\chi$ as expressed separates the action of the field of force, which affects $\sigma$, from the effect of density, which affects $l_{\mathrm{m}}$.

The problem discussed in this work essentially applies to dense media as compressed gases $\left(n_{1}>10^{20} \mathrm{~cm}^{-3}\right)$ discussed by Chapman and Cowling (1970) and some plasma matter such as the solar atmosphere $\left(n_{1}>10^{14} \mathrm{~cm}^{-3}, T \cong 10^{4} \mathrm{~K}\right)$. For rarefied collisionless plasmas in a strong magnetic field under the CGL (Chew, Goldberg, Low) approximation, the Larmor radius replaces the mean free path in the computation of $\chi$ (Schunk 1977). In this case, $\chi=\sigma \Omega / w_{\perp}$ where $\Omega$ is the cyclotron frequency and $w_{\perp}$ is the perpendicular component of the velocity respective to the magnetic field direction. Under these circumstances for the terrestrial equatorial electrojet $\sigma \cong \lambda_{\mathrm{D}} \cong 30 \mathrm{~cm}, \Omega=5 \times 10^{6} \mathrm{rad} \mathrm{s}^{-1}, w_{\perp}=72 \times 10^{7} \mathrm{~cm} \mathrm{~s}^{-1}$ and then $\chi \cong 0.2$. Hence, for this particular plasma instability phenomenon the proposed methodology applies. 
Despite the many theoretical efforts to date (see Balescu 1960; Baldwin 1962; Clemmov and Dougherty 1969; Liboff 1969; Chapman and Cowling 1970; Klimontovich 1982), no simple expression to effectively quantify and explain the modifications due to the increase of correlation in interactive gases and plasmas has been proposed. This is the purpose of the present work.

The presentation is organized starting from a proposed distribution function to describe the joint behavior of two interacting particles evolving to the actual effect of it on the macroscopic fluid equations. In Sec. 2 the joint distribution function is introduced. Section 3 presents its effects on the non-equilibrium equations. In Sec. 4 an analysis is made of the expected influence of the correlation for gases and plasmas. In Sec. 5 there is a discussion on the matter.

\section{The basic distribution function}

For rarefied gases and plasmas, Clemmov and Dougherty (1969) (following Klimontovich and Silin (1962)) suggested that the exact one particle distribution function of the mixture of particles could be described by

$$
F(\mathbf{r}, \mathbf{v}, t)=\sum_{i=1}^{N} \delta\left[R-R_{i}(t)\right]
$$

where $R$ is $(\mathbf{r}, \mathbf{v})$, a typical point of $\Gamma$ space (the phase space), and $R_{i}$ is the point relative to the $i$ th particle.

It is clear that this same expression holds for dense real gases and plasmas. These particles undergo a time evolution described by (1) and (2).

Selecting $n$ particles of the same species, an average one-particle distribution function is defined as

$$
f(\mathbf{r}, \mathbf{v}, t)=\left\langle F_{s}(\mathbf{r}, \mathbf{v}, t)\right\rangle
$$

where $\langle\cdot\rangle$ represents ensemble average and the subscripts specify the particle species. The function $f$ represents the number of particles within the volume $(\mathbf{r}, \mathbf{r}+d \mathbf{r})$ with velocity in the range $(\mathbf{v}, \mathbf{v}+d \mathbf{v})$ at time $t$. Any of these particles follows the time evolution given by (1) and (2).

A two-particle distribution function is also defined by

$$
f_{2}^{*}\left(\mathbf{r}, \mathbf{v}, \mathbf{r}_{1}, \mathbf{v}_{1}, t\right)+\delta\left(R-R_{1}\right) f(\mathbf{r}, \mathbf{v}, t)=\left\langle F_{\mathrm{s}}(\mathbf{r}, \mathbf{v}, t) F_{\mathrm{t}}\left(\mathbf{r}_{1}, \mathbf{v}_{1}, t\right)\right\rangle
$$

according to Clemmov and Dougherty (1969). The first term of the left-hand side (LHS) of (5) represents the joint distribution function of the two interacting particles outside the range of mutual influence, e.g. $f_{2}^{*}=f f_{1}$ (as in the Boltzmann term). The second term accounts for the joint behavior inside this range.

For a perfect gas, Landau and Lifchitz (1967) showed that under thermal equilibrium conditions the two particles distribution function for interacting particles has a Maxwellian shape $f_{0}$, with the reduced mass $\mu$ and relative velocity of the colliding particles. In addition, this distribution is consistent with the conservation of mass, momentum and energy during the encounter. For a real gas or plasma, small departures from equilibrium lead to distribution functions $f$, which slightly deviates from the Maxwellian form (Burgers 1969; Chapman and Cowling 1970; Schunk 1977; Zamlutti 1994) and will hereafter be called nearly Maxwellians. 
In the case of isotropic plasmas, the Navier Stokes distribution

$$
f=f_{0}(1+\Phi), \quad \text { with } \Phi=\frac{m}{2 k T p} \boldsymbol{\tau}: \mathbf{c c}-\left(1-\frac{m c^{2}}{5 k T}\right) \frac{m}{k T p} q \cdot \mathbf{c}
$$

is widely employed. Here $\mathbf{c}$ stands for the thermal velocity.

In order to produce a physically feasible reproduction of (5), we assume that outside the range of interaction the distribution function of the colliding particles are uncorrelated, e.g. $f_{2}^{*}=f f_{1}$, and inside that range they have an $f$-type shape with reduced mass and relative velocity (as in Landau and Lifchitz (1967)). This yields

$$
f_{2}=(1-\chi) H(x-\sigma) f(\mathbf{r}, \mathbf{v}, t) f_{1}\left(\mathbf{r}_{1}, \mathbf{v}_{1}, t\right)+\chi H(\sigma-x) f(\mathbf{x}, \mathbf{w}, t)
$$

where $\mathbf{x}=\mathbf{r}-\mathbf{r}_{1}$ and $\mathbf{w}=\mathbf{v}-\mathbf{v}_{1}$ are the relative distance and relative velocity, respectively, during a collision and $n_{1}$ is the target number density. Non-bold vector symbols represent their respective magnitudes. Here the distribution functions for the bullet and target particles (subscript 1) were assumed to be correlated and $\sigma$ is the magnitude of the radius of the sphere of interaction which surrounds the target (Liboff 1969; Cercignani 1988; Zamlutti 1994, 1999).

The use of complementary ranges of the configuration space adopted in $(6)$ is discussed in Sandri (1963). Other authors prefer not to establish the shape of the correlation function and replace $f(x, w, t)$ by a generic $g$ form (e.g. Frieman and Book 1963; Sandri 1963; Klimontovich 1982). They use $f_{2}=f_{2}^{*}+g_{2}$. Its proposal at this stage is justified grounded on the fact that during encounters, for a while, the bullet particle forces the target to behave identically to it, where the 'two body problem' is equivalent to a 'one body problem' in which one of the particles stays fixed and the other particle with fictitious mass $\mu$ describes a planar trajectory with respect to it.

The problem of continuity of the function $f_{2}$ is essentially related to the use of cutoff potentials (Cercignani 1967; Ordonez and Molina 1994) and will not be considered further here.

Next, we proceed with a test to verify whether the function $f_{2}$ proposed in (6) can in fact be accepted. First it must be consistent with the Boltzmann collision integral, for which ternary, quaternary, etc., encounters do not occur.

According to BBGKY theory (Montgomery and Tidman 1964; Liboff 1969) $f_{2}$ can only be accepted as satisfactory provided that:

(a) ternary, quaternary and higher order encounters are unlikely to occur;

(b) the bullet distribution function can be expanded in terms of the Maxwellian distribution function;

(c) the $\mathrm{BY}_{2}$ equation of the BBGKY hierarchy is satisfied (Liboff 1969).

For homogeneous media, the $\mathrm{BY}_{2}$ equation in the absence of external forces (Liboff 1969) under the above restrictions becomes

$$
\partial f_{2} / \partial t+\left(\mathbf{v} \cdot \nabla+\mathbf{v}_{1} \cdot \nabla_{1}\right) f_{2}-\left[\nabla U(\mathbf{x}) \cdot \nabla_{\mathrm{v}}+\nabla_{1} U(\mathbf{x}) \cdot \nabla_{\mathrm{v} 1}\right] f_{2}=0
$$

where $\nabla$ and $\nabla_{1}$ are the gradient operators for the bullet and target particles, respectively, $\nabla_{\mathrm{v}}$ and $\nabla_{\mathrm{v} 1}$ their gradient operators in velocity coordinates and $U(\mathbf{x})$ the central field potential which produces the interparticle force. Equation (7) actually expresses the closure of the BBGKY hierarchy with the essentials of 
the Boltzmann collision integral (namely condition (a)) employed in the theory of ideal gases. It is only reasonable in the transition from rarefied to dense gases and plasmas. With increasing density, condition (a) can no longer be maintained and some assumption as to the behavior of three particles distribution must be made. These results are in agreement with those by Frieman and Book (1963) and Klimontovich and Silin (1962).

Outside the sphere of radius $\sigma$ we recover from (7) a separate traditional force free collisionless Boltzmann equation for each particle. Inside the range of interaction, $\nabla_{1}=-\nabla$ and $(7)$ becomes

$$
\frac{\partial f}{\partial t}+\mathbf{w} \cdot \nabla f-\nabla U(\mathbf{x}) \cdot \nabla_{\mathrm{w}} f=0
$$

which is a collisionless Vlasov equation for $f(\mathbf{x}, \mathbf{w}, t)$ that holds if $f$ is a Maxwellianlike distribution. Thus (6) can be used as the effective joint distribution function to describe the behavior of binary elastic collisions, for rarefied, as well as for nearly dense, neutral and weakly ionized gases. It corresponds to the weak coupling approximation of Klimontovich (1982).

The presence of external forces just adds one more term to the square brackets of (7) (see Liboff 1969), but the same conclusion holds for nearly Maxwellian velocity distributions. When these forces drive vorticity fields peculiar situations appears (see Rostoker and Rosenbluth 1960).

The next step of the test is the computation of the Boltzmann collision integral as done in Zamlutti (1994) or Bird (1994).

Using the joint distribution function of $(6)$ in the collision integral, we get

$$
(\partial f)_{\mathrm{col}}=(1-\chi) \iint_{\sigma}^{\infty}\left(f_{1}^{\prime} f^{\prime}-f_{1} f\right)\left(\frac{d \mathbf{r}_{1}}{d t}\right) d \mathbf{v}_{1}+\chi \iint_{0}^{\sigma}\left[f^{\prime}(\mathbf{w})-f(\mathbf{w})\right]\left(\frac{d \mathbf{r}_{1}}{d t}\right) d \mathbf{v}_{1}
$$

where the details of $d \mathbf{r}_{1} / d t$ are given in Zamlutti (1994). The computation of the variations undergone during interactions are performed employing a Taylor series expansion as in Zamlutti (1999). The integration of $d \mathbf{r}_{1} / d t$ is performed in Zamlutti (1999). To first order (first term of the Fokker-Planck development) we obtain

$$
(\partial f)_{\mathrm{col}}=(1-\chi) \int w \mathbf{A}^{*} \cdot\left(\nabla_{\mathrm{v}}-\frac{m}{m_{1} \nabla_{v 1}}\right) f f_{1} d \mathbf{v}_{1}+\chi \int w \mathbf{A}^{*} \cdot \nabla_{\mathrm{v}} f(\mathbf{w}) d \mathbf{v}_{1}
$$

with

$$
\mathbf{A}^{*}=\left(\frac{\mu}{m}\right)\left(\mathbf{v}-\mathbf{v}_{1}\right) S_{1}=\left(\frac{\mu}{m}\right) \mathbf{w} S_{1}
$$

where $m$ stands for the bullet particle mass, $\mu$ for the reduced mass, $w$ for the magnitude of the relative velocity and $S_{1}$ for the cross section for momentum transfer (Zamlutti 1994, 1999).

The first nonlinear integral over the velocity space is carried out using $w_{0}=\langle w\rangle$ as an approach (see Zamlutti 1998) and recalling that the integral $\int \nabla_{\mathrm{vl}} \cdot\left[Q\left(\mathbf{v}_{1}\right) f_{1}\right]$ $d \mathbf{v}_{1}=0$ for any monomial $Q\left(\mathbf{v}_{1}\right)$ as demonstrated in Mintzer (1964). The second integral is computed using $w_{0}=\langle w\rangle$ and performing the integration over $\mathbf{v}_{1}$ using the distribution $f(\mathbf{w})=f\left(\mathbf{v}_{1}-\mathbf{v}\right)$. Then, we get

$$
(\delta f)_{\mathrm{col}}=(1-\chi) n_{1} w_{0} \mathbf{A} \cdot \nabla_{\mathrm{v}} f+\chi n_{1} w_{0} \mathbf{A} \cdot \nabla_{\mathrm{v}} f
$$


with $\mathbf{A}=(\mu / m)\left(\mathbf{v}-\left\langle\mathbf{v}_{1}\right\rangle\right) S_{1}$ and $f$ depending only on the incident particle velocity. It is clear that the $\chi$ terms cancel out in (8) and we recover the Boltzmann collision term. The collision term in (8) agrees with the result for $f_{2}=f f_{1}+g_{z}$ of Klimontovich (1982), for the case of the Boltzmann collision integral. In his notation, this last equation would be written as

$$
(\delta f)_{\mathrm{col}}=(1-\chi) n_{1} w_{0} \mathbf{A} \cdot \nabla_{\mathrm{v}} f+\chi n_{1} w_{0} \mathbf{A} \cdot \nabla_{\mathrm{v}} g_{2},
$$

which is sometimes helpful to remind us that it is the solution of the $\mathrm{BY}_{2}$ equation of Liboff (1969).

We can now look for the solution of the complete $\mathrm{BY}_{2}$ equation, when (7a) becomes

$$
\frac{\partial f^{*}}{\partial t}+\mathbf{w} \cdot \nabla f^{*}-\nabla U(x) \cdot \nabla_{\mathrm{w}} f^{*}=-n_{2}\left[\left(\frac{v}{k T}\right) \int \nabla U^{\prime} \mathrm{d} \mathbf{r}_{2}+\left(\frac{v_{1}}{k T_{1}}\right) \int \nabla U^{\prime \prime} d \mathbf{r}_{2}\right] f f_{1}
$$

where $n^{\prime}=\left(N_{2} / V\right)$, with $N_{2}$ the number of target particles of species 2 in the volume $V$. Here $U^{\prime}$ stands for this species potential with radius $\mathbf{x}^{\prime}=\mathbf{r}-\mathbf{r}_{2}$ and $U^{\prime \prime}$ that with radius $\mathbf{r}^{\prime}=\mathbf{r}_{1}-\mathbf{r}_{2}$.

A solution for (7b) will be considered later in this work (in the discussion) for the case $x^{\prime} \gg r^{\prime}$. The prospective distribution function will be $f^{*}=f_{0}(1+\Phi)(1+\Phi)$.

Before, we proceed it must be recalled that $\beta$ of (2) can be identified as $\beta=$ $(\mu / m)\left(m_{1} w_{0} S_{1}\right)$ using the momentum equation of Zamlutti (1994) and using the second term of the RHS of (8), the net effect of correlation is an effective variation of $\beta$ and $\mathbf{a}_{i}$ in (2) as indicated below:

$$
\begin{gathered}
\beta \rightarrow \beta-\chi\left(\frac{\mu}{m}\right)\left(n_{1} w_{0} S_{1}\right)=\beta(1-\chi) \\
\mathbf{a}_{i} \rightarrow \mathbf{a}_{i}-\chi\left(\frac{\mu}{m}\right)\left(n_{1} w_{0} S_{1}\right)\left(\mathbf{v}-\left\langle\mathbf{v}_{1}\right\rangle\right)=\mathbf{a}_{1}-\mathbf{a}_{i}^{*} .
\end{gathered}
$$

The reader may easy verify that results similar to $(9 \mathrm{a})$ and $(9 \mathrm{~b})$ would have been obtained if $h$ were replaced by $h^{*}$ in (2), as mentioned at the beginning of this work.

The actual consequence of the RHS of $(9 a)$ is that the distribution function computed with a weighted Boltzmann collision term (correlation aspects neglected) still holds as a dampening mechanism when correlations are considered.

The action of the complementary term in the RHS of $(9 \mathrm{~b})$ is to introduce a feedback mechanism whose product is an additive disturbance, $f^{*}$, on the distribution function, $f$, computed with the Boltzmann collision term. To obtain $f^{*}$, we can resort to the perturbation technique with the path integration method (see Clemmov and Dougherty 1969; Jensen and Oberman 1982).

The combined balance of the reduction of dampening due to (9a) with the development of perturbations produced by $(9 \mathrm{~b})$ is the growth of the turbulent dynamical behavior in fluids with the increase of its density. Of particular interest is the case of plasma micro-instabilities, for which an oscillatory driven excitation is able to survive in a dissipative medium. It corresponds to the well-known transition to chaos (Jensen 1987). 


\section{Effects of correlations on the equations}

Correlations affect fluid equations in a variety of forms depending or not on the presence of the magnetic or any other vorticity field action on $\mathbf{v}$. In the absence of these effects (neutral gases, plasma motion along field lines) the expected contribution is that of modifying the driving acceleration by an amount $\left\langle\mathbf{a}^{*}\right\rangle$ to be used in the LHS of the fluid equations (see Zamlutti 1994; Schunk 1977). As far as the RHS of the transport equations is concerned, its influence is that of an effective reduction in the collision frequency of the dampening term as stated by (9a). No significant modification of the results is expected relative to the computations using the traditional Boltzmann collision term to a first-order approximation, since the increase in density or temperature may compensate or even exceed the $(1-\chi)$ factor. So far its importance lies on the fact that it allows a better understanding of the inherent physical mechanisms going on during the interaction process.

In the presence of a magnetic field or any mechanism which produces a periodic behavior of $\mathbf{a}^{*}$, we face a different situation which corresponds to that of the standard map (Jensen and Oberman 1982) for the set of equations (1) and (2). It can be treated from a microscopic viewpoint as a perturbation, $f^{*}$, of the major distribution function. Under these circumstances the kinetic equation relating $f^{*}$ to $f$ becomes

$$
\left(\frac{d f^{*}}{d t}\right)_{u}=\mathbf{a}^{*} \cdot \nabla_{\mathrm{v}} f
$$

which also agrees with the formal solution for the correlation function suggested in Clemmov and Dougherty (1969). The time derivative follows an unperturbed orbit in the phase space. The path integral formalism is employed to determine $f^{*}$ (see Jensen and Oberman 1982). A discussion on this effect is presented in Rostoker and Rosenbluth (1960).

The macroscopic approach to handling the standard map is the perturbation method using the disturbing acceleration $\left\langle\mathbf{a}^{*}\right\rangle$ on the fluid equations. It is well described for the terrestrial ionosphere in Fejer et al. (1984).

Regardless of the methodology undertaken (microscopic or macroscopic) the net result of the presence of the standard map, to characterize the individual particles behavior, is the appearance of wavelike phenomena in fluids (Miyazaki and Adachi 1998; Sultan 1996).

\section{The effect of correlation}

As expected, for any sort of fluid, an increase in the density of target particles produces an increase in the importance of the incident particles (bullets) among the targets; the correlation term operates in the opposite sense. In fact, as shown by (9a) and $(9 \mathrm{~b})$, the correlation term transforms distributed reactions into a concentrated resistance force $\left(m \mathbf{a}^{*}\right)$ of a frictional nature attempting to stop the incident flow. Thus, it can be modeled in terms of a fictitious field (Vlasov equation in plasmas).

An interesting effect is produced by the action of external sources, responsible for the production of excited particles (Zamlutti 1997). These sources may drive large velocity differences, which compensate for a small $\chi$ and, according to $(9 \mathrm{~b})$, can be interpreted as field actions. This field following (10) drives microscopic perturbations. Depending on the energy involved, this effect can produce macroscopic 
disturbances or simply increases the turbulence of the medium. This aspect can be further explored by fusion physicists.

An example of an effect due to correlation occurs in the terrestrial equatorial ionosphere where the electric field which drives the so called 'equatorial electrojet' undergoes a reaction $\mathbf{a}^{*}$ following $(9 \mathrm{~b})$. The distributed field combined with the Earth's magnetic field generates plasma instabilities according to (10) (see Clemmov and Dougherty 1969). The Farley-Buneman instability results from this process and a secondary field is developed (whose value is given by $m \mathbf{a}^{*} / e$ ) opposite to the electrojet primary electric field (see Rogister 1971; Sato 1972).

\section{Discussion}

Kinetic theory is satisfactory for understanding the microscopic average behavior of individual particles of rarefied gases and plasmas. The collective behavior under similar conditions can be explained by fluid theory, (Serrin 1959) which is appropriate for dense media (Schunk 1983). The transition was never explored deeper, because apparently it would occur smoothly. The advent of the theory of chaos (see Ott (1981) for a review) and the need for kinetic transport theory (Stubbe 1990) to explain controversial transition problems called attention to a revision on the matter. This has been done in this work showing the importance of the correlation term in the joint two particles distribution during the collision time.

The subject was initially studied for plasmas where the long range of the Coulomb force invalidates the basic assumption of the Boltzmann collision term (Liboff 1969). The BBGKY kinetic theory was suggested (Rostoker and Rosenbluth 1960; Frieman and Book 1963; Montgomery and Tidman 1964; Liboff 1969) as an alternative approach to overcome the difficulty. It was further realized that the correlation contribution could be modeled in terms of a distributed field (Liboff 1969) and corresponds to the Vlasov term (LHS) of the kinetic equation describing the time evolution of the one particle distribution function.

A revision on the matter was presented in this work considering:

(a) a prospective form for the two particles joint distribution function;

(b) the influence of the correlation term considered as a perturbation;

(c) the effects of this term on kinetic and transport equations;

(d) the expected behavior of the gases and plasmas owing to the presence of correlation effects.

Regarding (a) above, two controversial aspects were presented:

(i) the range exclusion could introduce discontinuity at $x=\sigma$;

(ii) the a priori choice of the form $f$ for the correlation function could end up becoming unrealistic.

Although not critical, since $f_{2}$ only needs to be piecewise integrable, the first problem can be suppressed with the use of screened potential (see Ordonez and Molina 1994; Zamlutti 1999) and remotion of the Heaviside functions. In this case, we use $\exp -(x / \sigma) U(x)$ to replace $U(x)$ and drop $H(\sigma-x)$.

It is well known that the 'weak coupling approximation' (expressed here by (7a)) is equivalent to the binary collision approximation (Boltzmann or Fokker-Planck 
collision term) as determined by Frieman and Book (1963), Sandri (1963), and Klimontovich (1982). Under these circumstances, the correlation function $g$, can be expanded about an equilibrium state $f_{0}(\mathbf{x}, \mathbf{w}, t)$ and, in addition, the expansion yields a 13-moment or 16-moment distribution function depending on the isotropy or anisotropy of the medium (see Schunk 1977; Barakat and Schunk 1982; Zamlutti 1994). Thus the shape option at this stage is justified.

The correlation function using screened potentials (Zamlutti 1997, 1999) acquires the form

$$
g=\pi \sigma S_{1}^{*} f(\mathbf{w})
$$

where $S_{1}^{*}$ is the momentum transfer cross section for the screened potential (Zamlutti 1997, 1999) and $f(\mathbf{w})$ for isotropic media is

$$
\begin{aligned}
f= & f_{0}\left\{1+\rho\left(2 p^{2}\right)^{-1}\left\{\left(\boldsymbol{\tau}+\boldsymbol{\tau}_{1}\right):\left(\mathbf{c}_{1}-\mathbf{c}\right)\left(\mathbf{c}_{1}-\mathbf{c}\right)\right.\right. \\
& \left.\left.-2\left(\mathbf{q}_{1}-\mathbf{q}\right) \cdot\left(\mathbf{c}_{1}-\mathbf{c}\right)\left[1-0.2 \rho\left(\mathbf{c}_{1}-\mathbf{c}\right) \cdot\left(\mathbf{c}_{1}-\mathbf{c}\right)\right]\right\}\right\}
\end{aligned}
$$

with $\rho=n \mu, p=n k T, \boldsymbol{\tau}$ the stress tensor and $\mathbf{q}$ heat flow vector.

As for (b), the correlation term acts as a frictional reaction opposite to the driving force of the organized motion. This is expressed in $(9 \mathrm{~b})$ and allows us to interpret it as a perturbation $\mathbf{a}^{*}$ or a disturbance function $f^{*}$ related to $\mathbf{a}^{*}$ by (10).

With respect to (c), kinetic equations are affected by the presence of an additive term $-\mathbf{a}^{*} \cdot \nabla_{\mathrm{v}} f$ in the LHS of the Boltzmann equation. The transport equation is modified in its force term contribution as expressed in $(9 \mathrm{~b})$.

Concerning (d), a variety of situations may be analyzed. For dense gases, the Enskog theory (see Chapman and Cowling 1970) applies with their $\chi$ factor replaced by

$$
\chi^{*}=\left(1-\pi n_{1}^{*} \sigma^{3}\right)
$$

where the starred quantities represent compressed gases situations whereas the same unsuperscripted quantities denote STP conditions. For nearly dense gases, which correspond to the states of the terrestrial and solar atmosphere $\left(n \sim 10^{12}\right.$ $10^{14} \mathrm{~cm}^{-3}$ ), we expect that $\mathbf{a}^{*}$ just introduces a frictional reaction to the driving forces. In particular, in the terrestrial lower atmosphere it may introduce a feedback mechanism for externally driven localized wavelike excitation. In this case, if the driving energy is small the generated oscillation simply contributes to increasing the turbulence of the medium. For plasmas, the correlation term may be as important as the Boltzmann collision contribution because of a large $\chi$ and peculiar situations may arise from it (see Frieman and Book 1963; Zamlutti 1999).

Some authors have proposed correlation analysis based on the Enskog $\chi^{*}$ factor expressed by (13) as Dorfman and Cohen (1975), Van Beyeren and Ernst (1979), Garzo and Dufty (1999). Their derivations are quite involving, but the end results are equivalent to those of this work. The $\chi^{*}$ of $(13)$ is identical to our $(1-\chi)$ which weights the Boltzmann collision contribution. To account for our counter acceleration $\mathbf{a}^{*}$ they employed a restitution coefficient, which is only valid for soft spheres encounters and impact theory, and unsuitable for plasma studies.

Interesting situations involving the correlation term can arise in the case of partially ionized gases (such as those existing in the terrestrial upper atmosphere). Under certain conditions, charged-particle interactions are equivalent to the field action (correlation effect) while charged-neutral encounters introduce dissipation. The combined action of the two mechanisms can produce modifications in the 
behavior of electrojet plasma instabilities (Rogister 1971; Sato 1972; Dimant and Sudan 1995) and other phenomena characteristic of transition regions. The correlation term introduced here constitutes a bridge from rarefied to dense media. In fact, the development in series to improve $\mathbf{a}^{*}$ involves friction, viscosity and heat flow terms (Zamlutti 1998) when the density of neutral matter is increased to the point where correlation effects become appreciable.

As stated before, to a first-order approximation, only a small difference is expected in the present approach as compared with the traditional computations using the Boltzmann collision term. Its importance concerns the understanding of the mechanism, which reduces the influence of the organizing field actions observed in many nonlinear phenomena in the terrestrial upper atmosphere (e.g. Rogister 1971; Sato 1972).

The above results were expected since we employed the homogeneous $\mathrm{BY}_{2}$ instead of the complete $\mathrm{BY}_{2}$ equation. This modifies (7) to (7b), which has the form of the Boltzmann equation whose solution is the prospective form $f^{*}$ proposed before. The additional component due to simultaneous three particle interaction modifies (8) by the inclusion of a term due to

$$
\mathbf{a}^{* *}=\chi_{2}\left(n_{2} w_{0} \mathbf{A}^{* *}\right)
$$

with $\mathbf{A}^{* *}=\left(\mu^{*} / m\right)\left(\mathbf{w}-\left\langle\mathbf{v}_{2}\right\rangle\right) S_{2}$, where $\mu^{*}=\mu m_{2}\left(\mu+m_{2}\right)^{-1}$. Target-target interactions are assumed to not interfere in the process.

To conform with (14), the ternary correlation distribution function must be a Maxwellian with velocity $\mathbf{w}^{*}=\mathbf{w}-\left\langle\mathbf{v}_{2}\right\rangle$ and reduced mass $\mu^{*}$. Thus, with an increasing target medium density, the incident particle is continuously forced to move with the flow velocity of the target. To account for this effect, the secondorder distribution function becomes

$$
\begin{aligned}
f_{2}= & \left(1-\chi_{1}\right)\left(1-\chi_{1}\right) H\left(x_{1}-\sigma\right) H\left(x_{2}-\sigma\right) f(\mathbf{r}, \mathbf{v}, t) f_{1}\left(\mathbf{r}_{1}, \mathbf{v}_{1}, t\right) \\
& +\chi_{2} H\left(x_{1}-\sigma\right) H\left(\sigma-x_{2}\right) f\left(\mathbf{x}_{2}, \mathbf{w}, t\right) \chi_{1} H\left(\sigma-x_{1}\right) H\left(x_{2}-\sigma\right) f\left(\mathbf{x}_{1}, \mathbf{w}, t\right) \\
& -\chi_{1} \chi_{2} H\left(\sigma-x_{1}\right) H\left(\sigma-x_{2}\right) f\left(\mathbf{x}_{1}, \mathbf{w}^{*}, t\right) f\left(\mathbf{x}_{2}, \mathbf{w}^{*}, t\right)
\end{aligned}
$$

with $\mathbf{x}_{1}=\mathbf{r}-\mathbf{r}_{1}, \mathbf{x}_{2}=\mathbf{r}-\mathbf{r}_{2}$. This expression comprises the "polarization approximation' of Klimontovich (1982). Its effect on the equations of motion for identical target species will be given by the transformations

$$
\begin{gathered}
\beta \rightarrow \beta(1-\chi)^{2} \\
\mathbf{a}_{i} \rightarrow \mathbf{a}_{i}-2 \chi(\mu / m)\left(n_{1} w_{0} S_{1}\right)\left(\mathbf{v}-\left\langle\mathbf{v}_{1}\right\rangle\right)+\chi^{2}\left(\frac{\mu^{*}}{m}\right)\left(n_{1} w_{0} S_{1}\right)\left(\mathbf{w}-\left\langle\mathbf{v}_{1}\right\rangle\right) .
\end{gathered}
$$

In the general case of (15), the first-order approximation for the collision term is

$$
\begin{aligned}
(\delta f)_{\mathrm{col}}= & \left(1-\chi_{1}\right)\left(1-\chi_{2}\right) n_{1} w_{0} \mathbf{A}_{1} \cdot \nabla_{\mathrm{v}} f\left(\hat{\mathbf{w}}_{i}\right)+\chi_{1} n_{1} w_{0} \mathbf{A}_{1} \cdot \nabla_{\mathrm{v}} f\left(\hat{\mathbf{w}}_{1}\right) \\
& +\chi_{2} n_{2} w_{0} \mathbf{A}_{2} \cdot \nabla_{\mathrm{v}} f\left(\hat{\mathbf{w}}_{2}\right)+\chi_{1} \chi_{2} n_{2} w_{0} \mathbf{A}^{* *} \cdot \nabla_{\mathrm{v}} f\left(\hat{\mathbf{w}}_{1}-\left\langle\mathbf{v}_{2}\right\rangle\right)
\end{aligned}
$$

where the subscript numbers denote the target species. Also $\hat{\mathbf{w}}_{1}=\mathbf{v}-\left\langle\mathbf{v}_{1}\right\rangle, \hat{\mathbf{w}}_{2}=$ $\mathbf{v}-\left\langle\mathbf{v}_{2}\right\rangle$.

The proposed approach predicts that under the influence of correlation, the incident particle undergoes a drag by the colliding particle before actually being scattered. When $\chi=1$, no scatter occurs and the travelling particle must carry 
along all the targets that it finds in its trajectory. This is consistent with the field interpretation proposed in this work. To some extent, the present approach overcomes the major difficulties encountered by the Enskog method (see Chapman and Cowling 1970), namely inadequacy in the representation of physical reality and overestimation of corrective factors. The variation of the collision frequency with density and temperature is accounted for in the product $n_{1} w_{0}$ which affects $\beta$ (see Zamlutti 1994) and the correlation aspects are accounted for by $\chi$ as expressed by (9a). The Enskog factors do not effectively represent these two combined effects. It must be improved according to (13). In fact, the increase in the collision frequency with the density growth is due to the reduction of the free space between adjacent particles, whereas correlation aspects are related to similarities in the behavior of distinct particles, which improve with the enlargement of the radius of interaction.

\section{Summary for applications}

In order that regular users can benefit for the outcomes of the present work, we summarize as follows.

Consider the Boltzmann equation written as

$$
\frac{\partial f}{\partial t}+\mathbf{v} \cdot \nabla f+\mathbf{a} \cdot \nabla_{\mathrm{v}} f=(\delta f)_{\mathrm{col}}
$$

where $f, \mathbf{v}$, a are the distribution function, velocity and acceleration of the incident or bullet particle. This equation is valid for rarefied fluids.

The revised equation valid for dense fluids is

$$
\frac{\partial f}{\partial t}+\mathbf{v} \cdot \nabla f+\mathbf{a} \cdot \nabla_{\mathrm{v}} f-\sum_{i} \chi_{i} v_{i}\left(\mathbf{v}-\left\langle\mathbf{v}_{i}\right\rangle\right) \cdot \nabla_{\mathrm{v}} f\left(\hat{\mathbf{w}}_{i}\right)=\left[\prod_{i}\left(1-\chi_{i}\right)\right](\delta f)_{\mathrm{col}}
$$

where $\chi_{i}=\sigma_{i} / l_{\mathrm{m}}, \hat{\mathbf{w}}_{i}=\mathbf{v}-\left\langle\mathbf{v}_{i}\right\rangle, v_{i}=n_{i} w_{0} S_{i}$. Here $i$ denotes target particles species, $\langle\cdot\rangle$ ensemble average, $\sigma$ the radius of the sphere of interaction, $l_{\mathrm{m}}$ the mean free path, $w_{0}$ the relative thermal velocity, $S$ the cross section for momentum transfer, $n$ the number density and $v$ the collision frequency.

This last equation only accounts for linear effects. If only one target species exists, but is so dense that multiple collisions occur, scattering (RHS) becomes negligible and reactions to the driving forces (RHS) become stronger.

\section{Conclusions}

In this work, the basic aspects of the influence of the correlation term during collisional interaction on the kinetic and fluid equations were examined. It was shown that the expected effects range from a simple increase in turbulence to actual control of the studied phenomena. The intensity of the disturbance is related not only to the density of the medium, but also to the driving energy which controls the individual particle velocity. A probabilistic approach is necessary to account for the random aspect of the equations.

A particularly interesting outcome of the presented approach is that the correlated contribution can be interpreted and modeled as a field action.

The proposed theory is important for plasmas and partially ionized gases because of the long range nature of charged-charged encounters, as opposed to the short range effects of charged-neutral collisions. As for neutral gases, in addition to 
the traditional effects for compressed gases (see Chapman and Cowling 1970), an increase in turbulence may also be expected, either due to the large velocity of precipitating particles at the terrestrial auroral region according to (9b) or to the local wavelike excitation of an external nature at all latitudes.

The presented approach finds applications in the studies of the lower and upper terrestrial atmosphere, solar atmosphere, solar corona and solar wind, just to mention a few.

\section{Acknowledgements}

Support for this work came through Instituto Nacional de Pesquisas Espaciais, from FINEP under contract 537/CT. The author also received a complementary fellowship from Conselho Nacional de Desenvolvimento Científico e Tecnológico (CNPq) under the process 300901/90 (RN). The computer facility that helped in the preparation of the manuscript was provided by Fundação de Amparo à Pesquisa do Estado de São Paulo.

\section{References}

Baldwin, D. E. 1962 Close collisions in a plasma. Phys. Fluids 5, 1523-1530.

Balescu, R. 1960 Irreversible processes in ionized gases. Phys. Fluids 3, 62-63.

Barakat, A. R. and Schunk, R. W. 1982 Transport equations for multicomponent anisotropic space plasmas: a review. Plasma Phys. 24, 389-418.

Bird, G. A. 1994 In: Molecular Gas Dynamics and the Direct Simulation of Gas Flows. Oxford: Clarendon.

Burgers, J. M. 1969 Flow Equations for Composite Gases. Academic Press, New York.

Burghardt, I. and Cederbaum, L. S. 2001 Hydrodynamic equations for mixed quantum states. I. General formulation. J. Chem. Phys. 15, 10303-10311.

Cercignani, C. 1967 On Boltzmann equation with cutoff potentials. Phys. Fluids 10, 20972104.

Cercignani, C. 1988 The Boltzmann Equation and its Applications. Springer, New York.

Chapman, S. and Cowling, T. G. 1970 The Mathematical Theory of Non-uniform Gases. Cambridge: Cambridge University Press.

Clemmov, D. C. and Dougherty, J. P. 1969 Electrodynamics of Particles and Plasmas. Reading, MA: Addison-Wesley.

Collins, L. R. and Sundaram, S. 1998 Comment on 'Particle collision rate in fluid flows'. [Phys. Fluids 10, 1028 (1998)]; Phys. Fluids 10, 3247-3248.

Dimant, Y. S. and Sudan, R. N. 1995 Kinetic theory of low-frequency cross-field instability in a weakly ionized plasma. Phys. Plasmas 2, 1157-1168.

Dorfman, J. R. and Cohen, E. G. D. 1975 Velocity-correlation functions in two and three dimensions. II. Higher density. Phys. Rev. A 12, 292-316.

Fejer, B. G. Providakes and Farley, D. T. 1984 Theory of plasma waves in the auroral Eregion. J. Geophys. Res. 89, 7487-7494.

Forbes, J. M. and Garrett, H. B. 1979 Theoretical studies of atmospheric tides. Rev. Geophys. Space Phys. 17, 1951-1981.

Frieman, E. A. and Book, D. L. 1963 Convergent classical kinetic equation for a plasma. Phys. Fluids 6, 1700-1704.

Garzo, V. and Dufty, J. W. 1999 Dense fluid transport for inelastic hard spheres. Phys. Rev. E 39, 5895-5911.

Hirschfelder, J. O., Curtiss, C. F. and Bird, B. 1954 Molecular Theory of Gases and Liquids. New York: Wiley. 
Hu, K. C. and Mei, R. 1998 Particle collision rate in fluid flows. Phys. Fluids 10, 1028-1030.

Jensen, R. V. and Oberman, C. R. 1982 Statistical properties of chaotic dynamical systems which exhibit strange attractors. Physica D 4, 183-196.

Jensen, R. V. 1987 Classical chaos. Am. Sci. 75, 168-181.

Klimontovich, Y. L. and Silin, V. P. 1962 Theory of fluctuations of the particle distributions in a plasma. Soviet Phys.-JETP 15, 199-206.

Klimontovich, Y. L. 1982 Kinetic theory of nonideal gases and nonideal plasmas. Theory of Fluctuation of the Particle Distributions in a Plasma. New York: Pergamon Press.

Landau, L. and Lifchitz, E. 1967 Physique Statistique. Moscow: Ed. Mir.

Landau, L. and Lifchitz, E. 1996 Mécanique. Moscow: Ed. Mir.

Landau, L. and Lifchitz, E. 1971 Mécanique des fluides. Moscow: Ed. Mir.

Liboff, R. L. 1969 Introduction to the Theory of Kinetic Equations. New York: Wiley.

Lie-Svendsen, and Olsen, E. L. 1999 Comparison of kinetic and hydrodynamic descriptions of the proton polar wind in the transition to collisionless flow. J. Geophys. Res. 103, $4097-4113$.

Marsch, E. and Livi, S. 1987 Observational evidence for marginal stability of solar wind ion beams. J. Geophys. Res. 92, 7263-7268.

Mintzer, D. 1964 Transport theory of gases. In: Mathematics of Physics and Chemistry (ed. H. Marginau and G. M. Murphy). Princeton, NJ: Van Nostrand.

Miyazaki, T. and Adachi, K. 1998 Short-wavelength instabilities of waves in rotating stratified fluids. Phys. Fluids 10, 3168-3177.

Montgomery, D. C. and Tidman, D. A. 1964 Plasma Kinetic Theory. New York: McGraw-Hill.

Ordonez, C. A. and Molina, M. I. 1994 Evaluation of the Coulomb logarithm using cutoff and screened Coulomb interaction potentials. Phys. Plasmas 1, 2515-2518.

Ott, E. 1981 Strange attractors and chaotic motions of dynamical systems. Rev. Mod. Phys. 53, $655-671$.

Rogister, A. 1971 Nonlinear theory of 'type I' irregularities in the equatorial electrojet. J. Geophys. Res. 76, 7754-7760.

Rostoker, N. and Rosenbluth, M. N. 1960 Test particles in a completely ionized plasma. Phys. Fluids 3, 1-14.

Sandri, G. 1963 The foundations of nonequilibrium statistical mechanics, 1. Ann. Phys. 24, $332-379$.

Sato, T. 1972 Stabilization of the two-stream instability in the equatorial electrojet. Phys. Rev. Lett. 28, 732-734.

Schunk, R. W. 1977 Mathematical structure of transport equations for multispecies flow. Rev. Geophys. Space Phys. 15, 429-445.

Schunk, R. W. 1983 The terrestrial ionosphere. Solar-Terrestrial Physics (ed. R. L. Carovillano and J. M. Forbes). Reidel, pp. 609-676.

Serrin, J. 1959 Mathematical principles of classical fluid mechanics. In: Handbuch Der Physick, Band VIII/1. (ed. S. Flugge and C. Truesdell) Springer-Verlag, Berlin, 1959.

Sultan, P. I. 1996 Linear theory and modeling of the Rayleigh-Taylor instability leading to the occurrence of equatorial spread F. J. Geophys. Res. 101, 26 875-26 891.

Stubbe, P. 1990 The concept of a kinetic transports theory. Phys. Fluids B 2, 22-23.

Van Beyeren, H. and Ernst, M. H. 1979 Kinetic theory of hard spheres. J. Stat. Phys. 21, $125-167$.

Wan, C. and Schofield, J. 2000 Exact and asymptotic solutions of the mixed quantumclassical Liouville equation. J. Chem. Phys. 112, 4447-4459.

Xing, X. S. 1998 On the fundamental equation of nonequilibrium statistical physics. Int. J. Mod. Phys. B 12, 2005-2029.

Zamlutti, C. J. 1994 Transport equations for multicomponent isotropic and anisotropic high speed space plasmas. Planet. Space Sci. 42, 557-568. 
Zamlutti, C. J. 1997 On the effects of the reactive terms in the Boltzmann equation. Ann. Geophys. 15, 79-87.

Zamlutti, C. J. 1998 Elastic collision terms for transport equations of high flow velocity plasma. J. Phys. D 31, 712-716.

Zamlutti, C. J. 1999 Long range collision effects for drifting velocity distributions. Planet. Space Sci. 47, 57-66.

Zubarev, D. N., Morozov, V. G., Dmelyan, I. P. and Tokarchuk, M. V. 1991 Kinetic equations for dense gases and liquids. Plenum Publ. Corporation 412-424. 